\title{
Notas sobre los visitadores florales de Seemannia sylvatica (Kunth) Hanstein (Gesneriaceae)
}

\author{
Notes on floral visitors in Seemannia sylvatica (Kunth) Hanstein \\ (Gesneriaceae)
}

\section{Lianka Cairampoma y Carlos Martel}

Museo de Historia Natural-Universidad Nacional Mayor de San Marcos. Apartado 14-0434, Lima, Perú. E-mail: liankacb@gmail.com

Presentado: $\quad$ 05/05/2011 Aceptado: $\quad$ 23/07/2011

Publicado online: 02/10/2012

\begin{abstract}
Resumen
La polinización en Gesneriaceae comúnmente se asocia a síndromes de ornitofilia o melitofilia, siendo usual además la visita a las flores por especies robadoras de néctar. Se presenta una nota sobre la fauna que visita las flores de Seemannia sylvatica en Cusco, registrándose colibríes e insectos visitando las flores. En base a las características florales y conducta de los visitadores florales se sugiere que las flores de S. sylvatica presentan un síndrome ornitofílico. Se concluye que únicamente los colibríes serian los polinizadores, mientras que los insectos visitadores no intervendrían en la polinización y serian robadores del néctar de las flores.

Palabras clave: Síndrome floral, visitadores florales, polinización, Trochilidae, robadores de néctar.

\section{Abstract}

Pollination in Gesneriaceae commonly is associated with syndromes of ornithophily or melittophily, and usually others visitors are robbers of nectar. We present a note about the fauna that visit the flowers of Seemannia sylvatica in Cusco, where hummingbirds and insects were recorded visiting the flowers. Based on floral characteristics and behavior of floral visitors, we suggest that the flowers of S. sylvatica present an ornithophily syndrome. Therefore, only hummingbirds are pollinators, while the insects are not involved in pollination and only steal nectar from flowers.
\end{abstract}

Keywords: Floral syndrome, flower visitors, pollination, Trochilidae, nectar robbers

\section{Introducción}

La polinización puede ser llevada a cabo por distintos grupos funcionales de animales (Fenster et al. 2004), los cuales han sido clave en la diversificación de las angiospermas (Grant \& Grant 1965, Stebbins 1970, Fenster et al. 2004). Sin embargo, la relación planta-polinizador (mutualismo) puede ser afectada por los robadores florales (parasitismo), especies que no proveen servicios a las plantas (Maloof \& Inouye 2000).

Gesneriaceae es una de las familias tropicales más diversas, con 2500 a 3700 especies (Burtt \& Wiehler 1995). Son particularmente interesantes porque exhiben una alta variabilidad en sus formas florales y color (Skog 1976, Smith 1996), composición del néctar y asociación con diferentes síndromes de polinización (Perret et al. 2001).

La polinización por picaflores parece ser muy común y extendida en Gesneriaceae (Bawa et al. 1985, Perret et al. 2001). Se estima que alrededor del $60 \%$ de las Gesneriaceae neotropicales son ornitófilas, el 30\% melitófilas (específicamente abejas euglosas macho) y el $10 \%$ por el resto de otros grupos funcionales (Wiehler 1983). Son pocos los estudios en Gesneriaceae neotropicales enfocados en polinización y sistema reproductivo, y las tribus Episcieae, Gesnerieae y Sinningieae son las más estudiadas (Steiner 1985, San Martín-Gajardo \& Freitas 1999, Perret et al. 2001, San Martín-Gajardo \& Sazima 2004, 2005a, b, Martén-Rodríguez \& Fenster 2008, MarténRodríguez et al. 2009).

Seemannia sylvatica (Kunth) Hanstein, es herbácea terrestre, perenne con rizomas escamosos, numerosas flores por axila, no pedunculadas, sin brácteas, vistosas de corola tubular caracterizada por presentar tricomas multicelulares en forma de barril en la boca de la corola (Roalson et al. 2005); se distribuye en Bolivia, Brasil y Perú, forma densas poblaciones en causes de ríos, zonas abiertas de la llanura amazónica y bosques premontanos, y es ampliamente comercializada como especie ornamental (Wiehler 1976).

El propósito de esta investigación es (a) documentar los visitadores florales y (b) caracterizar la morfología y biología floral (longevidad floral, antesis, receptividad estigmática y dehiscencia de las anteras), y su evaluación en el contexto del sistema de polinización.

\section{Material y métodos}

Área de estudio. El presente estudio fue realizado en las cercanías del Albergue San Pedro, distrito Kosñipata, provincia de Paucartambo, departamento de Cusco, Perú (10,0556 S, $71,5466^{\circ} \mathrm{W}$ ), alrededor de los $1400 \mathrm{~m}$ de altitud, durante el mes de abril de 2009. El albergue está localizado en el flanco oriental de los Andes con una precipitación media anual de $2480 \mathrm{~mm}$ y una temperatura media de $15-18{ }^{\circ} \mathrm{C}$ (Atrium versión 1.7.1).

Morfología floral. Se hizo una adaptación de Navarro et al. (2007). Se tomaron medidas de distintas partes de la flor en individuos de $S$. sylvatica, longitud de la corola, diámetro de apertura de la corola, longitud del pistilo y de los estambres (Fig. 1). En total se tomaron las medidas de 75 flores (dos a tres por individuo). Con las medidas se realizó un análisis de componentes principales (ACP) para conocer si las variables morfométricas florales covarían. Los análisis morfométricos 




Figura 1. Variables morfométricas estudiadas en la flor de Seemannia sylvatica. 1 longitud de la corola; 2 diámetro de apertura de la corola; 3 longitud del estambre; 4 longitud del pistilo.

Tabla 2. Resultados del análisis de componentes principales con la matriz de variables florales en Seemannia sylvatica, indicando los valores de cada variable en los primeros dos componentes.

\begin{tabular}{lcc}
\hline Variables & PC I & PC II \\
\hline Longitud de corola & 0,0657 & $-0,3264$ \\
Diámetro apertura de corola & $-0,0579$ & 0,0365 \\
Longitud de pistilo & $-0,9523$ & $-0,2996$ \\
Longitud estambres & 0,2923 & $-0,8957$ \\
\% Varianza explicada & 68,306 & 22,039 \\
\hline
\end{tabular}

Tabla 3. Longitud del pico de los picaflores que visitaron las flores de Seemannia sylvatica.

\begin{tabular}{lc}
\hline Especie de Picaflor & Longitud del pico $(\mathbf{m m})^{*}$ \\
\hline Adelomyia melanogenys & 15 \\
Chaetocercus mulsant & 17 \\
Ocreatus underwoodii & 16 \\
Media de la corola de S. sylvatica & 18,1 \\
\hline
\end{tabular}

y los análisis de componentes principales fueron conducidos mediante el programa PAST versión 1.97 (Hammer et al. 2001).

Biología floral. Se monitoreó diariamente a individuos de $S$. sylvatica desde la apertura de la flor (día 1) hasta la pérdida de la corola (día 1+x). En total se utilizaron 75 flores. La receptividad estigmática fue examinada con el método de la actividad de la $\mathrm{H}_{2} \mathrm{O}_{2}$-catalasa (Zeisler 1938), desde el momento del "día 1" hasta el "día $1+x "$ ". La respuesta positiva a la prueba fue el burbujeo en una solución de $\mathrm{H}_{2} \mathrm{O}_{2}$. Se totalizaron 12 flores (una por individuo), por cada día de longevidad de la flor. Para conocer el momento de dehiscencia de las anteras se colectaron botones florales en diferentes estadios de desarrollo y se las diseccionó. Se observó el grado de madurez de las anteras y la morfología de los estambres hasta el último día de antesis de la flor.

Éxito de fructificación. Se marcaron y observaron 1135 flores para determinar la proporción que logra formar fruto. Luego de dos semanas de finalizada la antesis se colectaron los frutos y se observó si hubo desarrollo del mismo.

Visitadores florales. Las observaciones de los visitadores florales fue realizada en una temporada de floración de $S$. sylvatica en abril de 2009. Se realizaron 40 horas de observación entre las 6:00 y 17:00 horas (San Martín-Gajardo \& Freitas 1999). Se registró al visitador floral, la hora y duración de la visita. Los visitadores fueron colectados en etanol de $70 \%$ en caso de ser insectos, o mediante fotografías si eran aves.

\section{Resultados}

Morfología floral. Seemannia sylvatica presenta flores dispuestas en inflorescencias axilares o terminales, con 1-3 flores fasciculadas carentes de pedúnculos y con pedicelos teretes rojizos. Las flores son zigomorfas, erguidas con una corola tubular, sin olor perceptible, externamente anaranjado rojizo, internamente amarillo con puntos rojos en la base, y con notables glándulas en el interior de los lóbulos de la corola. Los estambres son exertos

Tabla 1. Medidas morfométricas florales tomadas en Seemannia sylvatica indicando los valores por día de antesis floral.

\begin{tabular}{lcccc}
\hline Longitud de estructuras $(\mathrm{mm})$ & Día $\mathbf{1}(\boldsymbol{\mu} \pm \mathrm{S})$ & Día $\mathbf{2}(\boldsymbol{\mu} \pm \mathrm{S})$ & Día $3(\boldsymbol{\mu} \pm \mathrm{S})$ & Día $\mathbf{4}(\boldsymbol{\mu} \pm \mathrm{S})$ \\
\hline Longitud de corola & $1,81 \pm 0,081$ & $1,83 \pm 0,108$ & $1,81 \pm 0,133$ & $1,76 \pm 0,121$ \\
Diámetro apertura máxima de corola & $0,599 \pm 0,086$ & $0,61 \pm 0,066$ & $0,63 \pm 0,085$ & $0,64 \pm 0,078$ \\
Longitud de pistilo & $1,43 \pm 0,213$ & $1,79 \pm 0,161$ & $1,77 \pm 0,402$ & $1,94 \pm 0,129$ \\
Longitud estambres & $1,44 \pm 0,106$ & $1,28 \pm 0,178$ & $1,12 \pm 0,149$ & $1,17 \pm 0,147$ \\
\hline
\end{tabular}




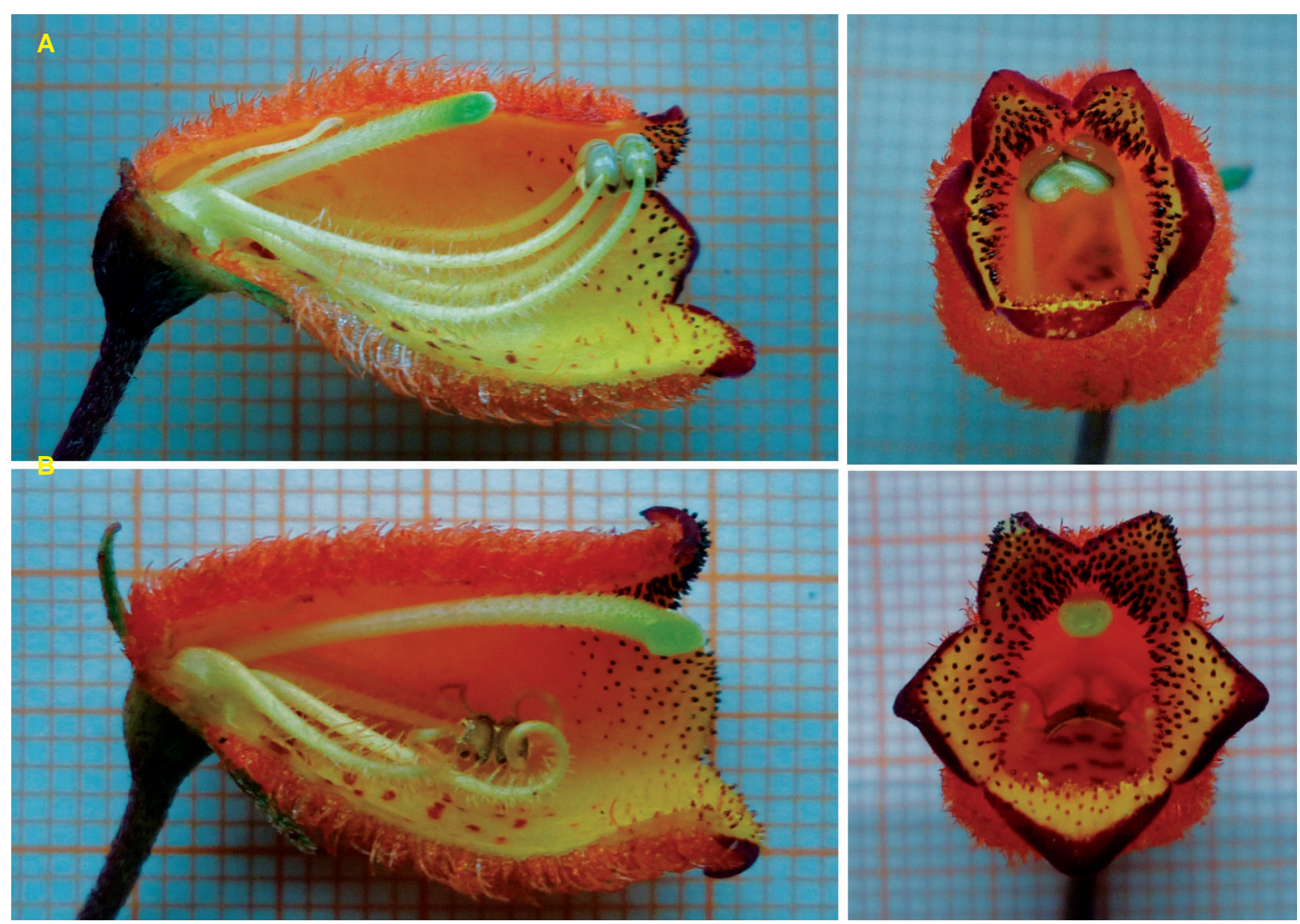

Figura 2. Vista longitudinal y frontal del desplazamiento de las estructuras reproductivas en diferentes fases sexuales (masculina y femenina) en flores de Seemannia sylvatica. A) Fase masculina, anteras expuestas y dehiscentes, estigma poco desarrollado; B) Fase femenina, anteras retorcidas sobre un lado de la corola y estigma bien desarrollado y expuesto.


Figura 3. Visitadores en Seemannia sylvatica. A) Adelomyia melanogenys (Trochilidae) alimentándose del néctar de una flor de S. sylvatica. B) Abeja Meliponini (Apidae) robando néctar a partir de un orifico en la base de la corola de S. sylvatica. 
Tabla 4. Número y proporción de flores robadas por día de antesis de Seemannia sylvatica

\begin{tabular}{|c|c|c|c|}
\hline Día & Flores robadas & $\%$ de flores afectadas & $\%$ diferencial de flores afectadas por día \\
\hline uno & $3(n=14)$ & 21 & 37 \\
\hline dos & $7(n=12)$ & 58 & 23 \\
\hline tres & $9(\mathrm{n}=11)$ & 82 & -10 \\
\hline cuatro & $5(\mathrm{n}=7)$ & 71 & \\
\hline
\end{tabular}

en número de 4; ovario ínfero con estilo incluido con presencia de un nectario anular en la base del mismo; estigma capitado y aplanado, exerto. (Fig. 1).

La media de la longitud del tubo de la corola fue $18,1 \pm 1,1$ $\mathrm{mm}(\mathrm{n}=69)$ y la media del diámetro de apertura $6,2 \pm 0,7 \mathrm{~mm}$ $(n=69)$. La longitud del gineceo se incrementó desde el día 1 $(10,6 \pm 1,3 \mathrm{~mm} ; \mathrm{n}=14)$ hasta el día 4 (último día de antesis, $15,3 \pm 1,1 \mathrm{~mm} ; \mathrm{n}=22)$. Los estambres disminuyeron de $14,4 \pm 1,1$ $\mathrm{mm}(\mathrm{n}=14)$ hasta $11,6 \pm 1,5 \mathrm{~mm}(\mathrm{n}=22)$. La variación de las longitudes por día se detalla en la Tabla 1.

$\mathrm{Al}$ realizar el ACP a partir de las variables florales, se observó que el primer componente explica el 68,31\% del total de la varianza en tanto que el segundo $22,04 \%$ (Tabla 2). La longitud del pistilo y longitud de estambres tienen los valores más altos en el primer componente, indicando que estas variables florales tienden a covariar más. El diámetro de apertura de corola es explicado por el segundo componente. Esto se refleja en el rápido desarrollo del pistilo y degeneración de los estambres que ocurre durante los días de antesis.

Biología floral. La duración de la antesis en todos los casos fue de 4 días ( $n=69$ flores). La dehiscencia de las anteras ocurrió al iniciarse la antesis (día 1) y el estigma alcanzó mayor receptividad los días 2 y 3. La flor se caracterizó por tener dos fases marcadas: Fase masculina, durante el primer día las anteras están dehiscentes y expuestas casi fuera de la corola. Fase femenina, a partir del tercer día, donde el estilo se ha elongado, y el estigma está más receptivo y se encuentra expuesto fuera de la corola. Los estambres a partir del tercer día se retraen (Fig. 2). El segundo día es una fase transicional entre la masculina y femenina. Por tanto, es una especie dicógama (protándrica) y hercógama (Faegri $\&$ van der Pijl 1979).

Visitadores florales. Se registró la visita de aves (Trochilidae) e insectos (Formicidae, Apidae y Coleoptera). Los visitadores más comunes fueron Adelomyia melanogenys (Fig. 3A), Chaetocercus mulsant y Ocreatus underwoodii (Trochilidae).

Se observó que durante las visitas a cada flor, el pico del picaflor hacía contacto con las anteras llenas de polen (flores en fase masculina) o con el estigma (flores en fase femenina). La longitud de los picos de los picaflores está indicada en la Tabla 3. Las visitas de los picaflores iniciaron temprano por la mañana y continuaron a lo largo del día hasta la tarde (desde las 8 hasta las 16 horas), con intervalos de 15 a 120 minutos entre cada visita. En promedio los Trochilidae visitaron las flores de $S$. sylvatica 37 veces por día, con una mayor frecuencia de visitas entre las 9 a 11 horas y visitando hasta 55 flores por vez.

Se observó una especie de Formicidae, una de Chrysomelidae, y una de Apidae (Trigona sp.), los que mostraron una conducta de robadores de néctar, al hacer o aprovechar forados en la base de la corola. Las Trigona sp. (ver Fig. 3B) hacían los orificios en la base de la corola, mientras las hormigas y escarabajos aprovechaban estos para consumir el néctar. Estas dos últimas especies tuvieron visitas esporádicas.

Se cuantificó la incidencia del robo de néctar a partir de la aparición de los orificios hechos por las abejas y su correspondencia con el día en que se hicieron (Tabla 4).

Éxito de Fructificación. Se contabilizaron 783 flores en las que luego se dio el desarrollo del fruto. El éxito total alcanzó el $68,97 \%$.

\section{Discusión}

Seemannia sylvatica presenta un síndrome de polinización ornitofílico (Faegri \& van der Pijl 1979, Proctor et al. 1996): forma tubular, posición horizontal a pendiente, color llamativo, olor no perceptible y néctar diluido. Las mismas que concuerdan con las características de sus visitadores florales, aves de la familia Trochilidae. Según la clasificación de Wiehler (1983), S. silvatyca sería del tipo 3 (corola urceolada, con un tubo marcadamente inflado, como una bolsa, y la boca muy constricta). Wiehler (1983) afirma que la bolsa, sirve para aumentar la visibilidad de la flor, mientras que la pequeña entrada asegura que las aves toquen las anteras y el estigma.

En S. sylvatica se observa una separación temporal y espacial de las anteras y el estigma. Esta separación del rol de los sexos dentro de una temporada reproductiva ocurre ampliamente en las plantas con flores, siendo encontrada en especies con flores perfectas en al menos 17 familias (Cruden 1988). Debido a que las flores de $S$. sylvatica presentaron hercogamia combinada con protandría, es poco probable que una autopolinización espontánea ocurra (autogamia). Posiblemente, esto es una estrategia para reducir la incidencia de autofecundación y de reducir el desperdicio de gametos, mediante el descuento en cantidades de polen (Lloyd \& Webb 1986). Esto debido a que la especie es autocompatible y capaz de desarrollar frutos con el polen en una misma flor (autogámica) (Cairampoma \& Martel, datos no publicados). También, podría ocurrir que en la misma planta existan flores en fase femenina y otras en fase masculina en un mismo momento, lo que podría ocasionar fertilizaciones geitonogámicas (flor polinizada con polen de otra flor pero que pertenece a la misma planta).

La relación morfológica entre la flor y el polinizador asegura la colocación del polen sobre el potencial polinizador. El tamaño de los picos de las especies Trochilidae visitadoras de S. sylvatica guardan relación con el tamaño de la corola $(18,1 \mathrm{~mm}$, Tabla 3), siendo siempre menores a esta longitud. Caso similar al registrado por San Martín-Gajardo \& Freitas (1999) en Besleria longimucronata. La menor longitud del pico de los Trochilidae que visitaron las flores de S. sylvatica aseguraba un contacto con las anteras y/o el estigma por lo que deducimos que Adelomyia melanogenys, Chaetocercus mulsant y Ocreatus underwoodii son sus 
polinizadores naturales. Aunque no podemos afirmar el impacto de cada especie de picaflor sobre la polinización de $S$. sylvatica.

El néctar es la recompensa más importante de las flores para los animales que las polinizan (Endress 1994, McDade $\&$ Weeks 2004). Camargo et al. (2011) registraron en $S$. sylvatica una producción de volumen de néctar floral de $6 \pm 4,9 \mu \mathrm{L}(\mathrm{n}=38)$, y

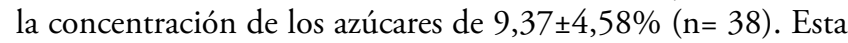
baja concentración del néctar coincidiría con las estrategias de otras plantas polinizadas por colibríes (Baker 1975, De la Barrera \& Nobel 2004). La baja concentración de néctar seria una estrategia para evitar el robo de néctar por parte de abejas (Bolten \& Feinsinger 1978); aunque, esto no es impedimento para la existencia de robadores de néctar de $S$. sylvatica como abejas, hormigas y escarabajos. Se observó que los robadores de néctar afectaron el 70 y 80\% (Tabla 4) hasta el cuarto día. Se observó que entre el día 1 y día 3, se dio el mayor porcentaje de robo (alrededor del 60\% del total). Esto podría explicarse porque el último día la planta disminuye su producción de néctar, al haber sido polinizada el día anterior (día 3). La tasa de robo sufrida por S. sylvatica es bastante elevada a pesar del néctar diluido.

El efecto de los robadores de néctar sobre el fitness de la planta es variable. En algunas especies es negativo, positivo o incluso neutro (Maloof \& Inouye 2000). En un sistema similar al estudiado, los robadores de néctar (ácaros) tuvieron un impacto positivo sobre el fitness de Moussonia deppeana, al incrementar el número de flores visitadas y la frecuencia de las visitas de sus colibríes polinizadores (Lara \& Ornelas 2002). Aunque no se puede aseverar lo mismo en S. sylvatica, sin un estudio respectivo. Cabe mencionar que los tres picaflores visitadores mostraron una conducta territorial. Lo que refuerza la idea de que los patrones de alimentación de los colibríes derivan en última instancia de las respuestas individuales a la disponibilidad de alimentos (Sazima et al. 1995, Sazima 1996). La competencia entre picaflores y el robo del néctar, favorecería un mayor número de visitas de los picaflores a distintas flores para copar sus requerimientos energéticos. Lo que se vería reflejado en la alta tasa de fructificación (cerca al 70\%). Aunque también podría causar una menor constancia de los picaflores a visitar las flores de S. sylvatica con poca o ninguna recompensa de néctar.

\section{Agradecimientos}

Agradecimiento muy especial a George Bradd por la ayuda en la identificación de los colibríes y el trabajo de campo. A las organizaciones IdeaWild, ACCA y TReeS-Perú por el apoyo con equipos y financiamiento del proyecto. Al lodge the Cock of the Rock por acogernos tan gentilmente.

\section{Literatura citada}

Atrium. 2005. Biodiversity Information System. Consultado en Noviembre 2009. Disponible en http://atrium.andesamazon.org.

Baker H.G. 1975. Sugar concentrations in nectars from hummingbird flowers. Biotropica 7(1): 37-41.

Bawa K.S., S.H. Bullock, D.R. Perry, R.E. Coville \& M.H. Grayum. 1985. Reproductive biology of tropical lowland rain forest trees. II. Pollination systems. American Journal of Botany 72(3): 346-356.

Bolten A.B. \& P. Feinsinger. 1978. Why do hummingbird flowers secrete dilute Nectar? Biotropica 10(4): 307-309.

Burtt B.L. \& H. Wiehler. 1995. Classification of the family. Gesneriaceae. Gesneriana 1: 1-4.
Camargo E., L. Rodrigues \& A. Araujo. 2011. Pollination biology and reproduction of Seemannia sylvatica (Kunth) Hanstein (Gesneriaceae) in the Serra da Bodoquena National Park, Mato Grosso do Sul. Biota Neotropica 11(4). Disponible en: http://www.biotaneotropica.org.br/v11n4/ en/abstract?article+bn02911042011

Cruden R.W. 1988. Temporal dioecism: systematic breadth, associated traits, and temporal patterns. Botanical Gazette 149: 1-15.

De la Barrera E. \& P.S. Nobel. 2004. Nectar: properties, floral aspects, and speculations on origin. Trends in Plant Science 9(2): 65-69.

Endress P. 1994. Diversity and Evolutionary Biology of Tropical Flowers. Cambridge: Cambridge University.

Faegri K. \& L. van der Pijl. 1979. The Principles of Pollination Ecology. 3rd edition. New York: Pergamon Press.

Fenster C.B., W.S. Armbruster, P. Wilson, J.D. Thomson \& M.R. Dudash. 2004. Pollination syndromes and floral Specialization. Annual Review of Ecology, Evolution and Systematics 35: 375-403.

Grant V., K.A. Grant. 1965. Flower pollination in the Phlox family. New York, NY, USA: Columbia University Press.

Hammer Ø., D.A.T. Harper \& P.D. Ryan. 2001. PAST: paleontological statistics software package for education and data analysis. Palaeont. Electr. 4: 1-9.

Lara C. \& J.F. Ornelas. 2002. Effects of nectar theft by flower mites on hummingbird behavior and the reproductive success of their host plant, Moussonia deppeana (Gesneriaceae). Oikos 96: 470-480.

Lloyd D.G. \& C.J. Webb. 1986. The avoidance of interference between the presentation of pollen and stigmas in angiosperms: I. Dichogamy. New Zealand Journal of Botany 24: $135-62$.

Maloof J.E. \& D.W. Inouye. 2000. Are nectar robbers cheaters or mutualists? Ecology 81(10): 2651-2661.

Martén-Rodríguez S. \& C.B. Fenster. 2008. Pollination ecology and breeding systems of five Gesneria species from Puerto Rico. Annals of Botany 103: 23-30.

Martén-Rodríguez S, Almarales-Castro A, Fenster CB. 2009. An evaluation of pollination syndromes in Antillean Gesneriaceae: evidence for bat, hummingbird and generalized flowers. Journal of Ecology 97: 348-359.

McDade L.A. \& J.A. Weeks. 2004. Nectar in hummingbirdpollinated Neotropical plants I: Patterns of production and variability in 12 species. Biotropica 36(2): 196-215.

Navarro L., G. Ayensa \& P. Guitia. 2007. Adaptation of floral traits and mating system to pollinator unpredictibility: the case of Disterigma stereophyllum (Ericaceae) in Southwestern Colombia. Pl. Syst. Evol. 266: 165-174.

Perret M., A. Chautems, R. Spichiger, M. Peixoto \& V. Savolainen. 2001. Nectar sugar composition in relation to pollination syndromes in Sinningieae (Gesneriaceae). Annals of Botany 87: 267-273.

Proctor M., P. Yeo \& A. Lack. 1996. The Natural History of Pollination. London: Harper Collins Publishers.

Roalson E.H., J.K. Boggan \& L.E. Skog. 2005. Reorganization of tribal and generic boundaries in the Gloxinieae (Gesneriaceae: Gesnerioideae) and the description of a new tribe in the Gesnerioideae, Sphaerorrhizeae. Selbyana 25(2): 225-238.

San Martín-Gajardo I. \& L. Freitas. 1999. Hummingbird pollination in Besleria longimucronata Hoehne (Gesneriaceae) in South-Eastern Brazil. Biociencias porto Alegre 7(2): 13-24.

San Martín-Gajardo I. \& M. Sazima. 2004. Non-Euglossine bees also function as pollinators of Sinningia species (Gesneriaceae) in southeastern Brazil. Plant Biology 6(4):506-512. 
San Martín-Gajardo I. \& M. Sazima. 2005 a. Espécies de Vanhouttea Lem. e Sinningia Nees (Gesneriaceae) polinizadas por beija-flores: interações relacionadas ao hábitat da planta e ao néctar. Revista Brasileira de Botânica 28(3): 441-450.

San Martín-Gajardo I. \& M. Sazima. 2005b. Chiropterophily in Sinningieae (Gesneriaceae): Sinningia brasiliensis and Paliavana prasinata are bat-pollinated, but P. sericiflora is not. Not yet? Annals of Botany 95(7):1097-1103.

Sazima I. 1996. An assemblage of hummingbird-pollinated flowers in a mountane forest in southeastern Brazil. Botanica Acta 109(2): 149-160.

Sazima I., S. Buzato \& M. Sazima. 1995. The saw-Billed hermit Ramphodon naevius and its flowers in southeastern Brazil. Journal fur Ornithologie 136(2): 195-206.

Schulenberg T., D. Stotz, D. Lane, J. O’Neill \& T. Parker III. 2007. Birds of Peru. Princenton University Press.

Skog L.E. 1976. A study of the tribe Gesnerieae with a revision of Gesneria (Gesneriaceae: Gesnerioideae). Smithsonian Contributions to Botany 29: 1-182.
Smith J.F. 1996. Tribal relationships within the Gesneriaceae: a cladistic analysis of morphological data. Systematic Botany 21: 497-513.

Stebbins G.L. 1970. Adaptive radiation of reproductive characteristics in angiosperms, I: Pollination mechanisms. Annual Review of Ecology and Systematics 1: 307-326.

Steiner K.E. 1985. The role of nectar and oil in the pollination of Drymonia serrulata (Gesneriaceae) by Epicharis Bees (Anthophoridae) in Panama. Biotropica 17(3):217-229

Wiehler H. 1976. A report on the classification of Achimenes, Eucodonia, Seemannia, Goyazia, and Anetanthus (Gesneriaceae). Selbyana 1:374-404

Wiehler H. 1983. A synopsis of the neotropical Gesneriaceae. Selbyana 6: 1-149.

Zeisler M. 1938. Über die Abgrenzung der eigentlichen Nabenfläche mit Hilfe von Reaktionen. Beihefte zum Botanisches Zentralblatt 58: 308-318. 